\title{
An Extreme Firm-Specific News Sentiment Asymmetry Based Trading Strategy
}

\author{
Qiang Song, Anqi Liu, Steve Y. Yang \\ Financial Engineering Department \\ Stevens Institute of Technology \\ Hoboken, New Jersey, USA \\ syang14@stevens.edu
}

\author{
Anil Deane, Kaushik Datta \\ Civil Finance Group \\ Northrop Grumman Information Systems \\ McLean, VA, USA \\ anil.deane@ngc.com
}

\begin{abstract}
News sentiment has been empirically observed to have impact on financial market returns. In this study, we investigate firm-specific news from the Thomson Reuters News Analytics data from 2003 to 2014 and propose an optimal trading strategy based on a sentiment shock score and a sentiment trend score which measure extreme positive and negative sentiment levels for individual stocks. The intuition behind this approach is that the impact of events that generate extreme investor sentiment changes tends to have long and lasting effects to market movement and hence provides better prediction to market returns. We document that there exists an optimal signal region for both indicators. And we also show extreme positive sentiment provides better a signal than extreme negative sentiment, which presents an asymmetric market behavior in terms of news sentiment impact. The backtest results show that extreme positive sentiment generates robust and superior trading signals in all market conditions, and its risk-adjusted returns significantly outperform the S\&P 500 index over the same time period.
\end{abstract}

Keywords - News Sentiment; Thomson Reuters News Analytics; Extreme Sentiment Shock and Sentiment Trend; Trading Strategy.

\section{INTRODUCTION}

Many studies have demonstrated that news media can affect financial markets and often becomes drivers of market activities [1]-[6]. Analyzing news contents and translating them into trading signals have become an attractive research topic in both academia and industry. There have been a number of studies that further document the value of using media sentiment to make trading decisions [9]-[11]. The motivation of this study is based on recent findings that news content affects investor sentiment and market volatility [5], [7], [8]. We propose a trading strategy based on extreme news sentiment levels on individual stocks, and we further explore the effect of a long and short strategy based on extreme positive and negative sentiment on these stocks.

In a previous study, we showed that not all news has the same impact on an investor's sentiment toward financial markets, and the abnormal shocks of news sentiment can provide us better signals in predicting cumulative market return and implied volatility of the S\&P 500 index [12]. For this study, we utilize the same trend and shock indicators to capture abnormal patterns of firm-specific news sentiment and generate trading signals for individual stocks. We first filter company stocks by the news frequency to ensure that their sentiment movements follow a consistent statistical pattern. The design of our trading strategy is based on the hypothesis that the rankings of news sentiment of a set of firms reflect the relative confidence of investors toward these firms, and hence their relative market performance in the near future. The primary contribution of this paper is to document that a longonly strategy based on extreme positive sentiment shocks and trend can generate robust trading signals and consequently outperform the buy-and-hold S\&P500 index strategy during both high volatility and low volatility market conditions. Furthermore, we investigate the inconsistency of predictive power according to different sentiment polarities. Several studies have tested the hypothesis that there exists an asymmetric market reaction to news. For example, investors underreact to the official news (e.g. earning announcements) and overreact to a series of good or bad news [2]. Smales pointed out that in the Gold future market, the negative news has superior impacts on volatility to positive sentiment [13]. Generally, negative news is more informative and therefore the reaction to it is more vigorous [14]. By comparing the long-only and short-only strategies, we demonstrate that extreme positive sentiment is a more reliable signal to predict potential increasing of stock prices than extreme negative sentiment. This presents a reversal effect of previous findings.

The rest of the paper is structured as the following: We first review the existing literature about financial news and market reactions as well as the existing techniques in exploiting these relationships in section II. In section III, we discuss the Thomson Reuters News Analytics data and present the aggregated sentiment and market return data used in this study. We then formulate the two extreme sentiment scores and propose a long-short strategy framework in section IV. We perform the strategy parameter optimization and discuss the backtest results in section V. Finally, we conclude the findings and highlight contributions of this study in section VI.

\section{BACKGROUND AND LITERATURE REVIEW}

\section{A. Financial News Impacts to Market Activity}

In general, research on financial news impact on market activities has been targeted to answer two primary questions: 1) Does the news information lead to financial market 
activities or price movements? 2) Can special patterns of news sentiment form indicators that reliably predict subsequent market price or volatility changes?

A number of studies have provided sufficient empirical evidence that there exist statistical relationships between news and financial markets. Three types of news influences have been investigated for different market features. First, news information is associated with subsequent market return. By analyzing millions of messages from Yahoo! Finance and Raging Bull, Antweiler and Frank documented that the number of posts has significant correlation with market return [4]. Second, financial news helps to predict market volatility. In our previous study, a linear regression model has been developed to demonstrate that the abnormal news sentiment has significant prediction power to the implied volatility of S\&P 500 index in the following few days [12]. Third, news sentiment impacts trading volume. Ahmad et al. conducted sentiment analysis on firm-specific news and concluded that the sentiment is related to stock trading volume [16]. Antweiler and Frank discussed this question from the view point of disagreement in news and confirmed that fluctuations of sentiment polarity raise trading volume [4].

\section{B. Trading Strategies using News Sentiment}

The findings of news impact on financial market has led to further studies of designing algorithmic strategies based on news sentiment. Tetlock applied firm-specific news content of a previous trading day in a trading strategy and concluded that the negative content in media information provides significant predictive power in risk-adjusted returns [17]. In a similar study, Nassirtoussi et al. implemented a multi-layer dimension reduction algorithm on news headlines to predict the intraday direction of the USD-EUR pair and achieved an accuracy of $83 \%$ [18]. Mitra incorporated both market information and news sentiment in estimating equity portfolio volatility [19]. In another study, Healy designed a real-time news analytics framework and used Thomson Reuters News Scope data to manage investment risks and returns [20]. Leinweber justified the predictability of news sentiment to market returns and designed sentiment based portfolio strategies [21].

\section{DATA}

We use market data and financial news sentiment data from Bloomberg terminal and Thomson Reuters News Analytics respectively. The data range is from January 2003 to December 2014.

\section{A. Market data}

The market data with stock price and trading volume are obtained from Bloomberg terminal. Returns are calculated to match the range of the news sentiment data. To design a trading strategy, we need to first define a stock universe, and stocks that are out of this universe will not be considered in trading. In this study, we only include stocks that are traded in the US market. The stock universe is selected with two steps: the first step is to select stocks with high liquidity (as a result, the top 1000 stocks with highest average trading volume are selected, and ETFs, are removed); the second step is to filter out stocks with too few news reports (stocks on average with less than one news article per week are excluded from the trading universe). The resulting stock universe includes 596 stocks. Fig. 1 shows the GICS (Global Industry Classification Standard) sectors of the selected stocks. Comparing with S\&P 500 index, we show that our selected stock universe is a good approximation of the large-cap market.

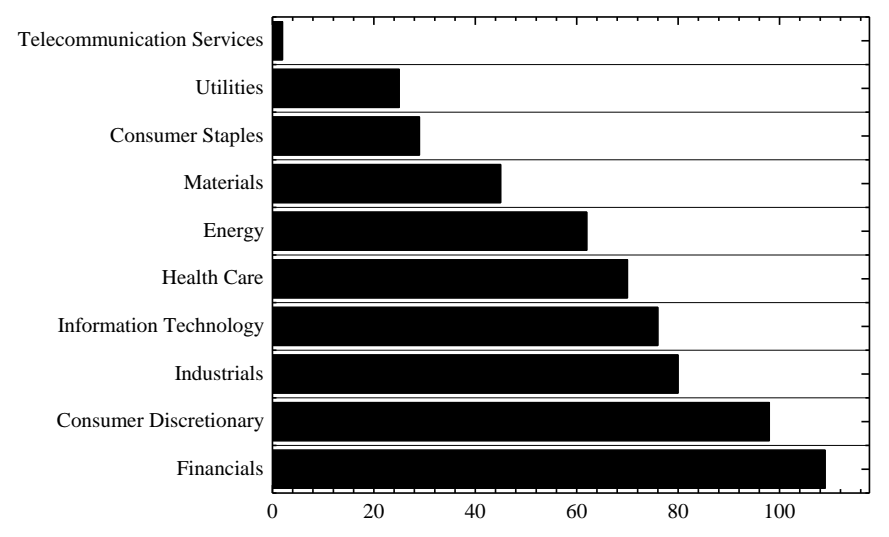

Fig. 1. Number of Stocks by GICS sectors.

\section{B. News Senetiment data}

Thomson Reuters is a major financial data provider. Thomson Reuters News Analytics automatically analyzes news and provides quantified insight into the events in the news, and its numerical form can be directly used by automated trading systems. The whole data range is from January 2003 to December 2014. There are more than 80 metadata fields in the Thomson Reuters News Analytics, and the fields listed below are used in this study.

Date/Time: The date and time of the news article.

Stock RIC: Reuters Instrument Code (RIC) of the stock for which the sentiment scores apply.

Sentiment Classification: A integer number indicate the predominant sentiment value for a news with respect to a stock identified by the RIC. Possible values are 1 for positive sentiment, 0 for neutral and -1 for negative sentiment.

Sent_POS: Positive Sentiment Probability, the probability that the sentiment of the news article is positive for the stock. The possible value ranges from 0 to 1 .

Sent_NEUT: Neutral Sentiment Probability, the probability that the sentiment of the news article is neutral for the stock. The possible value ranges from 0 to 1 .

Sent_NEG: Negative Sentiment Probability, the probability that the sentiment of the news article is negative for the stock. The possible value ranges from 0 to 1 . The sum of the three probabilities (Sent_POS, Sent_NEUT, Sent_NEG) equals 1.

Relevance: A real-valued number between 0 and 1 indicating the relevance of the news item to a stock. A single news article may refer to multiple stocks, by comparing the 
number of occurrences within the text, the stock with the most mentions will be assigned with the highest relevance, a stock with a lower number of mentions will have a lower relevance value.

In order to calculate a sentiment score for each stock mentioned in one news item, we first calculate the expected value of the sentiment score, and then generate the weighted expected value using its relevance value. Finally, the weighted weekly average sentiment score is calculated as follows:

$$
\text { Avg_Sent }=\frac{1}{N} \sum_{N} \begin{gathered}
\left(\left(\text { Sent_POS }_{\mathrm{N}} \times 1\right.\right. \\
+ \text { Sent_NEUT } \times 0 \\
+ \text { Sent_NEG } \times(-1)) \\
\times \text { Relevance }),
\end{gathered}
$$

where $N$ is the total number of new articles for a stock within one week. The scores are computed weekly and incorporate news on business days, weekends and holidays from Monday to Sunday each week. The weighted weekly average sentiment is later used as the input for computing the other two sentiment scores.

\section{Summary Statistics of Sentiment Data}

Table I shows the summary statistics of the news sentiment data, and it includes the mean, standard deviation, maximum, minimum, and 5th, 50th, and 95th percentile of each variable. The top row shows the calculated average sentiment for each stock; the bottom row shows the number of news articles for each stock.

TABle I. Satistics of Calculated Average Sentiment

\begin{tabular}{|c|ccccccc|}
\hline \multicolumn{2}{|c}{ MEAN } & STD. & MAX & MIN & $5 \%$ & $50 \%$ & $95 \%$ \\
\hline \multirow{2}{*}{ Avg.SENTIMENT } & 0.09 & 0.24 & 0.83 & -0.78 & -0.20 & 0.00 & 0.60 \\
& & & & & & & \\
No. OF NEWS & 5.18 & 11.69 & 830 & 0 & 0 & 2 & 22 \\
\hline
\end{tabular}

In Fig. 2, we plot the monthly aggregated average news sentiment for all 596 stock, the total number of news articles (hereinafter "number of news") for each month, and the S\&P 500 index monthly return. As shown in the chart, the average news sentiment is positively correlated with market return with a correlation coefficient of 0.21 , the total number of news is negatively correlates with market return with a correlation coefficient of -0.14 . Through lead-lag analysis, the news sentiment is also shown to lead the market return, but market return has no effect on the future news sentiment.

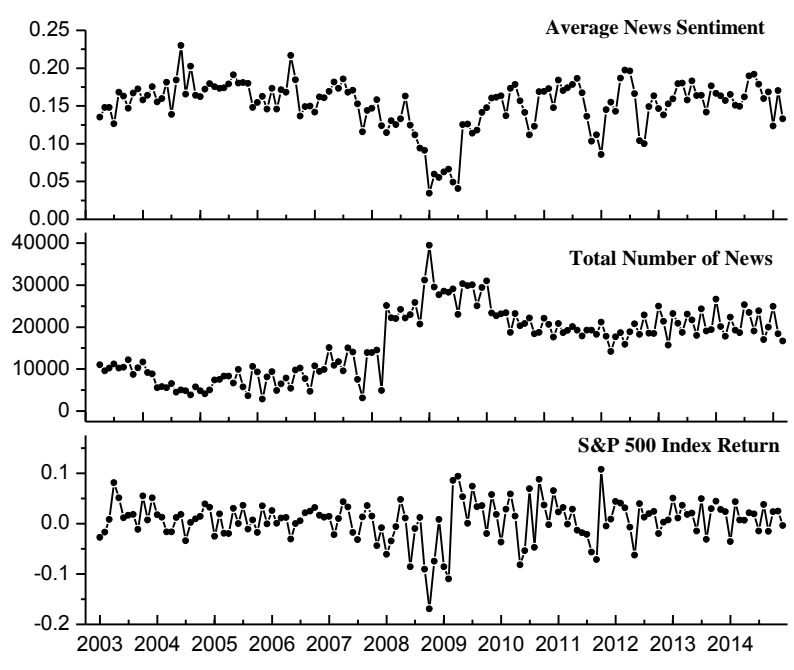

Fig. 2. Monthly Aggregated News Data Comparing with Market Returns

\section{TRAding Strategy DESIGN AND OPTIMIZATION}

News sentiment has been shown to have significant impact on the financial market, but how to use the sentiment to design a trading system is not straightforward. In this study, we take advantage of the Thomson Reuters New Analytics data and explore the abnormal levels of the news sentiment data. We propose two sentiment scores to characterize shocks (i.e. spike up or down) and trends in the sentiment time series. The calculation is based on the weekly average news sentiment scores for each stock. In order to reduce the number of parameters in the trading strategy and avoid over-fitting, we optimize the calculation parameters for each GICS sector. All stocks within the same sector use the same parameter. The trading strategy is designed to monitor the calculated sentiment scores and generate buy-and-sell signals for each stock.

\section{A. Sentiment Shock and Trend Scores}

The time series data of news sentiment, as shown in the top chart of Fig. 2, may not directly correlate with the return of an individual stock. For example, the absolute value of the sentiment scores is not a direct predictor for the magnitude of future stock returns. We need to look into the structure of the time series and consider both the current sentiment value and previous values. Here we propose to two sentiment scores: a sentiment shock score and a sentiment trend score to characterize the shocks and trend in the news sentiment time series data.

Sentiment shocks are spikes observed from the time series plot, these sentiment shocks are often caused by the release of unexpected macroeconomic data, financial report results, and corporate actions. The sentiment shock score is calculated as below:

$$
\left(S_{t 0}-\mu\right) / \sigma
$$


where $S_{t 0}$ is sentiment value on week $t 0$, to represents the current week, $\mu$ is the mean of sentiment values from week $t 0$ $N$ to $t 0-1$, and $\sigma$ is the standard deviation of sentiment values from week $t 0-N$ to $t 0-1 . N$ is the total number of look-back weeks.

The sentiment trend score is the aggregated change of sentiment or the sum of deltas of sentiment levels. The change of sentiment may be more informative than the absolute sentiment levels, especially after being aggregated over a period of time. This kind of sentiment trend may be caused by a series of good news or bad news, and this may have a strong impact on investor sentiment and future asset prices. The sentiment trend score is calculated as below:

$$
\sum_{i=t 0-N}^{t 0} \Delta S_{i}
$$

where $\Delta S_{i}$ is the change of sentiment in week $i$, and $t 0$ represents the current week. $N$ is the moving window size, summing the change of sentiment within it.

\section{B. Parameters Optimization}

Each of the sentiment shock or trend score has a parameter $N$ (the look-back window) to choose. To find the best parameter, we use Spearman rank correlation as the objective value. In order to reduce the number of parameters and avoid over-fitting, $N$ is optimized for each GISC sector, and stocks in the same sector use the same value. The method we use to optimize these parameters is to maximize the Spearman rank correlation between the sentiment scores and the next week's stock return. The Spearman rank correlation is a measure of rank dependence between two variables. For a sample of size $n$, the two variable $X_{i}, Y_{i}$ are converted to ranks $x_{i}, y_{i}$, the correlation coefficient is computed as:

$$
\rho=1-\frac{6 \sum d_{i}^{2}}{n\left(n^{2}-1\right)}
$$

where $d_{i}=x_{i}-y_{i}$. By maximizing the rank correlation, the calculated sentiment scores are most informative for future stock return.

\section{Trading Strategy}

Using the optimized look-back windows for each sector, we then construct the time series of sentiment shock and sentiment trend scores for each stock from 2003 to 2014. The design of our trading strategy is based on the hypothesis that extreme sentiment has a persistent effect on subsequent stock returns. Therefore, the trading process is to long stocks with extremely high positive sentiment scores or short stocks with extremely low negative sentiment scores.

\section{1) Extreme Sentiment Threshold}

The cutoff of extreme sentiment score versus regular score range is defined according to the probability distribution of sentiment scores during the training period (see Fig. 3). A threshold of bottom 5\% means the sentiment score in the $5 \%$ bottom percentile in the training data is the break point of extreme negative sentiment, and scores lower than that threshold are considered as short signals. As it shows in Fig. 3, the majority of the sentiment scores are centered around zero. This means, as we choose larger thresholds, the sentiment value diminishes quickly and only the extreme values are of significance in providing signals. This effect is particularly pronounced in the sentiment trend score, as it has a broader non-zero region in the cumulative distribution function than that in the sentiment shock score. We will further discuss the optimal selection criterion for this threshold next.
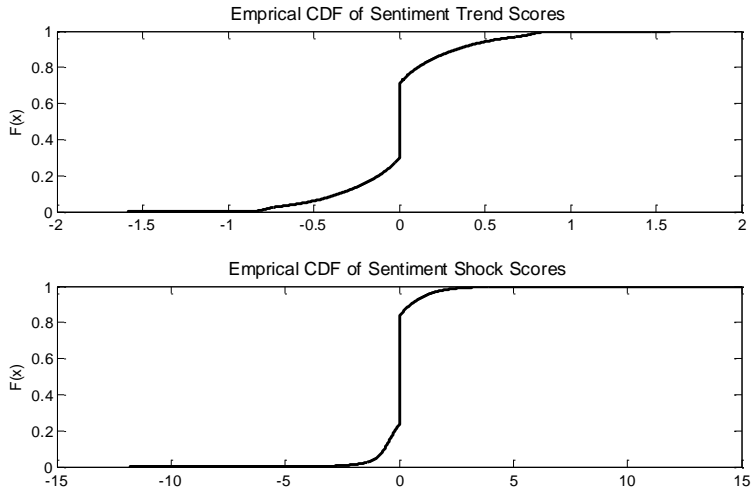

Fig. 3. Empirical cumulative distribution functions (CDFs) of sentiment trend scores and sentiment shock socres.

\section{2) Trading Procedure}

We design a dynamic trading framework with weekly evaluations and monthly re-adjustments. We determine the threshold of extreme sentiment, for example, $90 \%$ as positive threshold and $10 \%$ as negative threshold. Each firm-specific sentiment score is evaluated and compared with the threshold to make trading decision. If the firm's sentiment exceeds (or below) the positive (or negative) threshold, we long (or short) its stock. The default position re-adjustment is scheduled every 4 weeks. To avoid severe loss before re-adjusting, we set a stop loss limit of $10 \%$ drop. In the trading system, the return is recorded every week and the re-adjustment process is enforced as long as the loss exceeds $-10 \%$ (see Fig. 4).

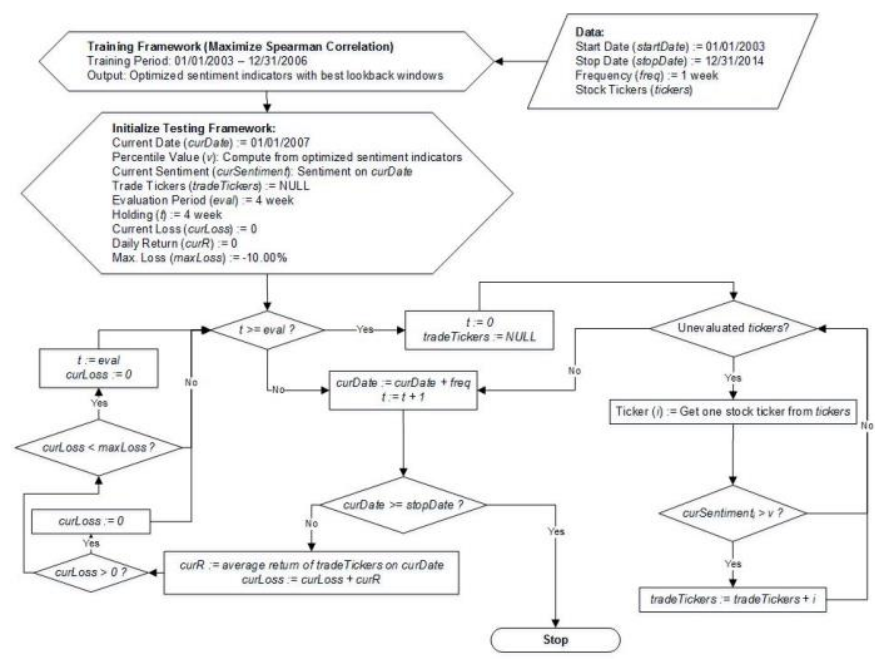

Fig. 4. Trading Strategy Diagram (Long Strategy) 


\section{RESUltS AND DisCUSSION}

In this section, we discuss the parameter optimization results and backtest results using the framework and the trading strategy defined in the previous section. To optimize parameters, we use 4 years of data from 2003 to 2006 so that we can test how the strategies perform during the 2008 financial crisis. TABLE II. summarizes the optimized lookback windows of sentiment indicators for each sector.

TABLE II. OPTIMIZED NUMBER OF WEEKS FOR SENTIMENT SCORES

\begin{tabular}{|c|c|c|}
\hline Sector Name & Sentiment Shock & Sentiment Trend \\
\hline Consumer Discretionary & 15 & 14 \\
\hline Information Technology & 11 & 30 \\
\hline Consumer Staples & 18 & 19 \\
\hline Materials & 15 & 16 \\
\hline Industrials & 21 & 18 \\
\hline Utilities & 16 & 28 \\
\hline Health Care & 10 & 15 \\
\hline Energy & 25 & 20 \\
\hline Financials & 11 & 25 \\
\hline Telecommunication Services & 19 & 24 \\
\hline
\end{tabular}

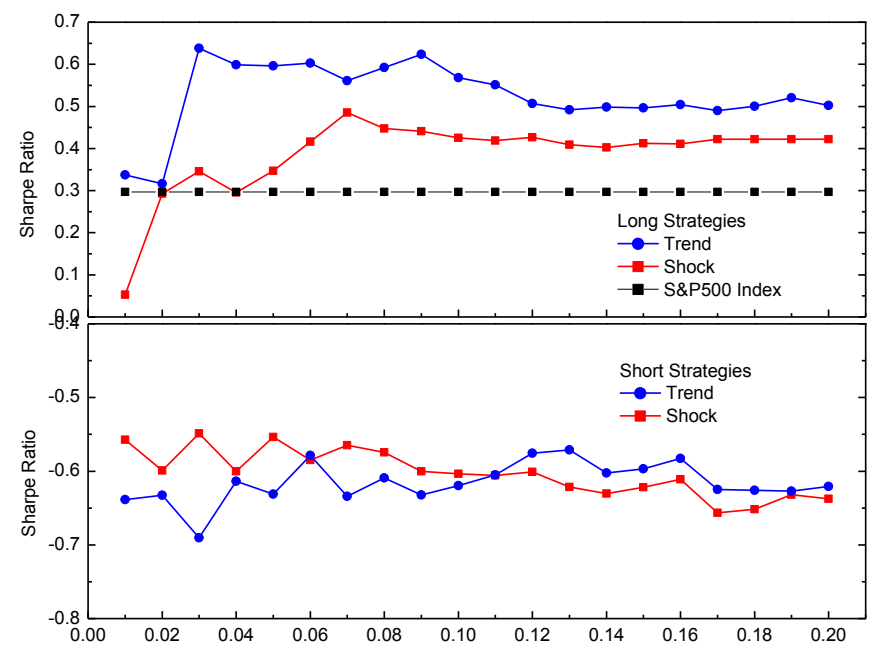

Fig. 5. Strategy Sharpe ratios by changing extreme sentiment selection percentile. Top chart shows long strategy with threshold from top $1 \%$ to $20 \%$ and bottom chart shows short strategy with threshold from bottom $1 \%$ to $20 \%$.

We backtested both long and short trading strategies using sentiment shock and trend scores from 2007 to 2014. To obtain the optimal extreme sentiment threshold, we tested the cutoff ranges from top $1 \%$ to top $20 \%$ and bottom $1 \%$ to bottom $20 \%$ for long and short strategy respectively. The cutoff score is determined by the sentiment distribution during the training period (see Fig. 3). For the long strategy, both shock indicator and trend indicator have better Sharpe ratios than the S\&P500 (see Fig. 5). In addition, as we increase the cutoff percentile, the Sharpe ratio rises to a peak and gradually flattens out. This phenomenon can be explained from the following two perspectives. 1) When the cutoff percentile rises, more stocks are added into the trading portfolio. The Sharpe ratio increases in the first stage because more companies with superior returns are included for better diversification. 2) The following decrease of Sharpe ratio is primarily caused by the diminishing effect of the news influence, and it plateaus when there is a little market impact that can be added into the portfolio. Moreover, the trend indicator strategy performs better than the shock indicator strategy in terms of higher Sharpe ratio in every cutoff percentile. More interestingly, we find there is a range of cutoffs for the extreme sentiment trend indicator that yield optimal risk-adjusted returns, while there is only one peak cutoff that exists for the extreme sentiment shock indicator. Opposite to the good performance of long strategies, the short strategies result in negative Sharpe ratios. The different results for long and short strategies demonstrate the asymmetric response of the market to extreme positive and negative sentiment. Thus we will not include the short strategies in the following performance measures.

In order to test the robustness of the trading strategies, we recorded the trading activities for each strategy (Table III). For both sentiment indicators, the number of winning trades almost doubles the number of losing trades. This indicates that the consistent performance of the strategy, and the good results are not from several lucky trades.

\section{TABLE III. BACKTEST STATISTICS FOR LONG STRATEGIES}

\begin{tabular}{|c|c|c|c|c|c|}
\hline Strategy & $\begin{array}{c}\text { Max. } \\
\text { Drawdown }\end{array}$ & $\begin{array}{c}\text { No. of } \\
\text { Trades }\end{array}$ & $\begin{array}{c}\text { No. of } \\
\text { Wining } \\
\text { Trades }\end{array}$ & $\begin{array}{c}\text { No. of } \\
\text { Losing } \\
\text { Trades }\end{array}$ & $\begin{array}{c}\text { Avg. } \\
\text { Holding } \\
\text { Period } \\
\text { (Weeks) }\end{array}$ \\
\hline $\begin{array}{c}\text { Sentiment } \\
\text { Trend }\end{array}$ & $49.09 \%$ & 673 & 449 & 224 & 9.51 \\
\hline $\begin{array}{c}\text { Sentiment } \\
\text { Shock }\end{array}$ & $56.37 \%$ & 896 & 576 & 320 & 6.96 \\
\hline
\end{tabular}

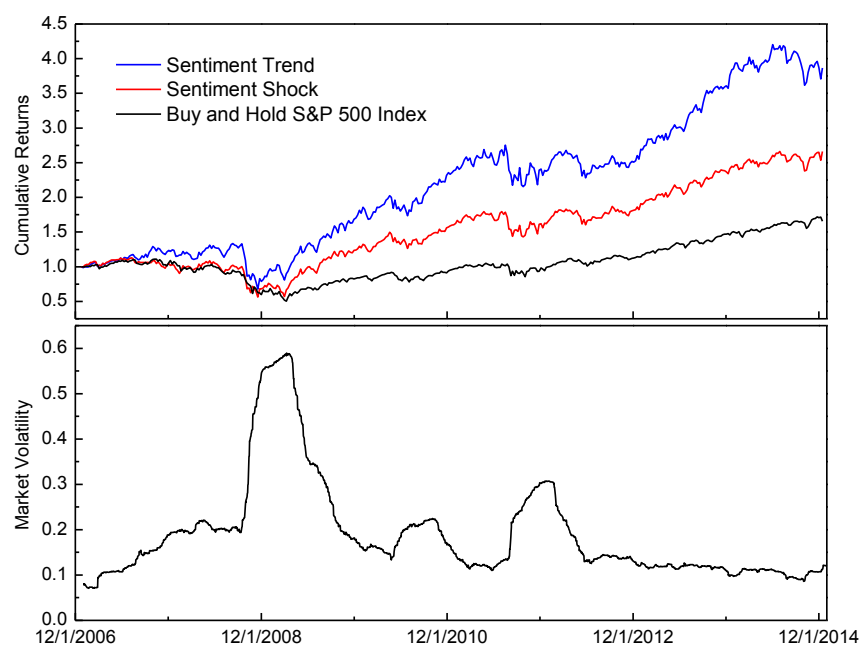

Fig. 6. Cumulative returns of Sentiment Trend and Shock strategy benckmarked with Buy and Hold S\&P500 Index. Top chart shows the Long strategy, bottom chart shows the market volatilty for the same time peroid.

The top chart of Fig.6 shows the cumulative returns of our trading strategies, the result from the buy-and-hold strategy of S\&P 500 index is also included as a benchmark, the bottom 
chart of Fig. 3 shows the market volatility. As shown in the top chart, the long strategies outperform the buy-and-hold strategy for the whole test period, and this also confirms that sentiment trend trading strategy has better performance than sentiment shock. In addition, to further test the performance of strategies in different market conditions, we split the backtest period into high volatility regime and low volatility regime using 6-month realized market volatility (see bottom chart in Fig. 6). We set the threshold as two standard deviations larger than the average, which is $36.93 \%$. The high volatility regime during 2003 to 2014 was from 10/2008 to 05/2009. Both sentiment indicator strategies show higher profitability than the benchmark strategy in high volatility regime. In the low volatility regime that was bull market period, the trend indicator outperform the benchmark in terms of higher return and Sharpe ratio. The shock indicator exhibits the same level of performance compared with the buy-and-hold strategy with a slightly lower Sharpe ratio (see TABLE IV. ). This result demonstrates that both sentiment indicators have good performance in predicting subsequent market returns in the long run, and the sentiment trend indicator provides more robust trading signals than the sentiment shock indicator. As shown in Table III, the long strategies using the extreme positive sentiment outperform S\&P 500 index in both high and low market volatility regimes.

TABLE IV. BACKTEST RESUltS IN DIFFERENT MARKET CONDITIONS

\begin{tabular}{|c|c|c|c|}
\hline \multirow{2}{*}{ Strategy } & \multicolumn{3}{|c|}{ Annualized Performance Measures } \\
\cline { 2 - 4 } & Mean Return & Volatility & Sharpe Ratio \\
\hline \multicolumn{4}{|c|}{ Total Backtest Period } \\
\hline Sentiment Trend & $16.90 \%$ & $26.50 \%$ & 0.64 \\
\hline Sentiment Shock & $12.24 \%$ & $25.22 \%$ & 0.49 \\
\hline Buy\&Hold S\&P 500 & $6.30 \%$ & $21.18 \%$ & 0.30 \\
\hline \multicolumn{4}{|c|}{ High Volatility Regime } \\
\hline Sentiment Trend & $56.40 \%$ & $52.60 \%$ & 1.07 \\
\hline Sentiment Shock & $49.21 \%$ & $54.28 \%$ & 0.91 \\
\hline Buy\&Hold S\&P 500 & $-19.94 \%$ & $47.92 \%$ & -0.42 \\
\hline \multicolumn{4}{|c}{ Low Volatility Regime } \\
\hline Sentiment Trend & $13.61 \%$ & $23.11 \%$ & 0.59 \\
\hline Sentiment Shock & $9.15 \%$ & $21.18 \%$ & 0.43 \\
\hline Buy\&Hold S\&P 500 & $8.49 \%$ & $17.29 \%$ & 0.49 \\
\hline
\end{tabular}

\section{CONCLUSION}

In this study, we use firm-specific news data from Thomson Reuters News Analytics, and we propose a sentiment shock score and a sentiment trend score for individual stocks to identify extreme sentiment levels and consequently used them as trading signals. A previous study has shown that abnormal news sentiment, like sentiment shocks and trends, are predictive for future market return and volatility [12]. We show that at individual stock level, the same intuition still applies, viz. that a big jump of the sentiment or a trend of sentiment change in the same direction will trigger persistent impact on stock price movement. In order to reduce the number of parameters in the trading strategy design to avoid over-fitting, we optimized the parameters for each GICS sector, stocks that are in the same sector use the same parameters. The parameters are selected so that the cross-sectional ranks of the sentiment scores are most aligned with the ranks of futures returns. Based on the empirical distribution of the sentiment scores, we designed a long and a short trading strategy. Long stocks with sentiment scores fall in the top percentiles, or short stocks with sentiment in the bottom percentiles. Transaction costs and market impacts are not considered in our experiments. We only use buy-and-hold strategy in the S\&P 500 market as the benchmark. There are a number of ways to measure transaction costs to assess the absolute performance of a trading strategy. These issues will be further studied in the future work.

The backtest results demonstrate that extreme positive sentiment can generate robust trading signals in both high volatility and low volatility regimes, and the performance of long-only strategy is superior to buy-and-hold S\&P 500 in terms of mean return and Sharpe ratio, but the short strategy using extreme negative sentiment underperforms the benchmark in all market conditions. Our results show that the extreme positive sentiment for individual stocks generates more reliable trading signals than the extreme negative sentiment. This is also an indication of the asymmetric response of the market to positive and negative sentiment. This finding seems contradictory to Tetlock's earlier finding that firm-specific negative content has reliable prediction to returns [17]. However, we believe it might be the effect of behavior reversal under the extreme circumstances. This is out of the scope of this paper, but this will be a new topic for future research.

\section{REFERENCES}

[1] M. Mitchell, M. Mitchell, H. Mulherin, and H. Mulherin, "The Impact of Public Information on the Stock Market," J. Finance, vol. 49, no. 3, pp. 923-950, 1994.

[2] N. Barberis, A. Shleifer, and R. Vishny, "A model of investor sentiment," J. financ. econ., vol. 49, no. 3, pp. 307-343, Sep. 1998.

[3] L. Mitra and G. Mitra, The Handbook of News Analytics in Finance. Chichester, West Sussex, UK: John Wiley \& Sons, Ltd., 2011.

[4] W. Antweiler and M. Z. Frank, "Is All That Talk Just Noise? The Information Content of Internet Stock Message Boards," J. Finance, vol. 59, no. 3, pp. 1259-1294, Jun. 2004.

[5] B. M. Barber and T. Odean, "All That Glitters: The Effect of Attention and News on the Buying Behavior 
of Individual and Institutional Investors," Rev. Financ. Stud., vol. 21, no. 2, pp. 785-818, Dec. 2007.

[6] J. Scott, M. Stumpp, and P. Xu, "News, not trading volume, builds momentum," Financ. Anal. J., vol. 59, no. 2, pp. 45-54, 2003.

[7] D. DiBartolomeo and S. Warrick, Linear Factor Models in Finance. Elsevier, 2005.

[8] P. C. Tetlock, M. Saar-Tsechansky, and S. MacSkassy, "More Than Words: Quantifying Language to Measure Firms' Fundamentals," J. Finance, vol. 63, no. 3, pp. 1437-1467, Jun. 2008.

[9] W. Zhang and S. Skiena, "Trading Strategies to Exploit Blog and News Sentiment.," Icwsm, vol. 34, pp. 375-378, 2010.

[10] S. F. Crone and C. Koeppel, "Predicting exchange rates with sentiment indicators: An empirical evaluation using text mining and multilayer perceptrons," in 2014 IEEE Conference on Computational Intelligence for Financial Engineering \& Economics (CIFEr), 2014, pp. 114-121.

[11] M. Kaya, "Stock price prediction using financial news articles," Inf. Financ. Eng. ICIFE 2010 2nd IEEE Int. Conf., pp. 478-482, 2010.

[12] S. Y. Yang, Q. Song, S. Yin, K. Mo, K. Datta, and A. Deane, "The Impact of Abnormal News Sentiment on Financial Markets," J. Bus. Econ., vol. Accepted, 2015 .

[13] L. a. Smales, "Asymmetric volatility response to news sentiment in gold futures," J. Int. Financ. Mark. Institutions Money, vol. 34, pp. 161-172, Jan. 2015.

[14] R. Riordan, A. Storkenmaier, M. Wagener, and S. Sarah Zhang, "Public information arrival: Price discovery and liquidity in electronic limit order markets," J. Bank. Financ., vol. 37, no. 4, pp. 11481159, Apr. 2013.

S. Yin, K. Mo, A. Liu, and S. Y. Yang, "News Sentiment to Market Impact and its Feedback Effect," SSRN Working Paper, Apr. 2015.

[16] K. Ahmad, C. Kearney, and S. Liu, "No news is good news: A time-varying story of how firm-specific textual sentiment drives firm-level performance," Eur. Financ. Manag. May, 2013.

[17] Tetlock, "Giving Content to Investor Sentiment: The Role of Media in the Stock Market," J. Finance, vol. 62, no. 3, pp. 1139-1168, Jun. 2007.

[18] A. Khadjeh Nassirtoussi, S. Aghabozorgi, T. Ying Wah, and D. C. L. Ngo, "Text mining of newsheadlines for FOREX market prediction: A Multilayer Dimension Reduction Algorithm with semantics and sentiment," Expert Syst. Appl., vol. 42, no. 1, pp. 306-324, Jan. 2015.

[19] L. Mitra, G. Mitra, D. Dibartolomeoll, and D. Dibartolomeo, "Equity portfolio risk estimation using market information and sentiment," Quant. Financ., vol. 9, no. 8, pp. 887-895, Dec. 2009.

[20] A. D. Healy and A. W. Lo, "Managing real-time risks and returns: The Thomson Reuters NewsScope event indicies. Handbook of News Analytics in Finance." John Wiley \& Sons, 2011.

[21] D. Leinweber and J. Sisk, Relating news Analytics to Stock Returns. Handbook of News Analytics in Finance.” John Wiley \& Sons, 2011. 